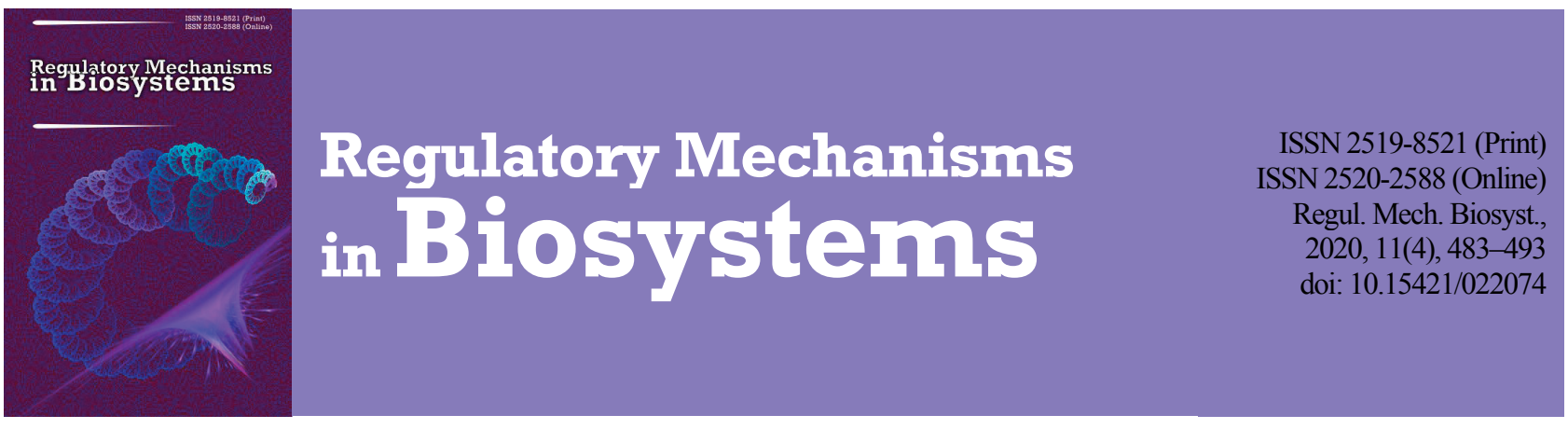

\title{
Regulation of redox processes in biological systems with the participation of the Keap1/Nrf2/ARE signaling pathway, biogenic selenium nanoparticles as Nrf2 activators
}

\author{
V. S. Bityutsky*, S. I. Tsekhmistrenko*, O. S. Tsekhmistrenko*, N. O. Tymoshok**, M. Y. Spivak** \\ *Bila Tserkva National Agrarian University, Bila Tserkva, Ukraine \\ **D. K. Zabolotny Institute of Microbiology and Virology of the National Academy of Sciences of Ukraine, Kyiv, Ukraine
}

Article info

Received 18.10.2020

Received in revised form 10.11.2020

Accepted 11.11.2020

Bila Tserkva National Agrarian University

Soborna Sq., 8/1,

Bila Tserkva,

09117, Ukraine.

Tel.: +38-096-374-71-51.

E-mail:voseb@ukr.net

D. K. Zabolotny Institute

of Microbiology and

Virology of the National

Academy of Sciences

of Ukraine,

acad. Zabolotnyst., 154

Kyiv, 03680, Ukraine.

Tel.: +38-063-45-46-385. Email:ntimoshok@ukr.net

Bityutsky, V. S., Tsekhmistrenko, S. I., Tsekhmistrenko, O. S., Tymoshok, N. O., Spivak, M. Y. (2020). Regulation of redox processes in biological systems with the participation of the Keap1 / Nrf2 / ARE signaling pathway, biogenic selenium nanoparticles as Nrf2 activators. Regulatory Mechanisms in Biosystems, 11(4), 483-493. doi:10.15421/022074

The article is devoted to the mechanisms of regulation of redox processes in cells, a review of the Keap1 / Nrf2 / ARE redoxsensitive signaling system as a fundamental pathway that plays a key role in maintaining cellular redox homeostasis under stressful, inflammatory, carcinogenic and proapoptotic conditions. The structure of the cysteine-rich repressor protein Keapl, which is responsible for sensory perception of electrophiles and reactive oxygen species, the structure and functions of the transcription factor Nrf2, mechanisms of Nrf2 activation through the Keap1 / Nrf2 / ARE signaling system, which regulates the transcription and expression of cellular cytoprotective and antioxidant proteins, are described. Published data on the specificity of the interaction of the components of this cellular signaling pathway, the mechanisms of Keap1 dependent and independent adaptive response to the action of inductors, the role of biogenic selenium nanoparticles synthesized by green chemistry with the participation of bacteria in these processes are analyzed; features of Nrf2 induction depending on the type of bacteria and the stabilizing shell. It has been shown that biogenic selenium nanoparticles (BNSe), synthesized by different types of bacteria, activate the transcription factor Nrf2 using the Keap1independent activation pathway through mitogen-protein kinases (MAPK): p38, ERK1 / 2 and AKT-mediated phosphorylation of $\mathrm{Nrf}$, protect the intestinal epithelial barrier function from the effects of oxidative damage, normalize mitochondrial function. A detailed understanding of thiol-dependent and independent redox signaling mechanisms under physiological and pathological conditions will lead to a deeper understanding of the redox component in human and animal diseases. The use of biogenic nanoselen, synthesized with the participation of various bacterial species, has been demonstrated to activate the Keap1 / Nrf2 / ARE signaling pathway, which may be of practical interest as a therapeutic target for many redox-mediated diseases.

Keywords: redox processes; reactive oxygen species; nanotechnology; "green chemistry"; biogenic nanoselen; cytoprotective proteins; antioxidant proteins.

\section{Introduction}

Redox reactions are essential for life as key transducers of cell signaling and metabolism. The relationship and interdependence of redox processes in the cell at different spatial and temporal scales remain poorly understood (Held et al., 2020). Cellular redox systems include a wide range of components that carry out cooperative intersecting signaling (Yoshida et al., 2016), possessing a certain hierarchy with different characteristics and kinetics. They include five main categories (Held et al., 2020).

1. Low-molecular-weight redox pairs (NADPH-NADP ${ }^{+}, \mathrm{NADH}_{-}$ $\mathrm{NAD}^{+}$, reduced glutathione-oxidized glutathione), usually catalytically bound to antioxidant proteins.

2. Antioxidant proteins: peroxiredoxins, thioredoxin (TRX) and glutathione-utilizing enzymes.

3. Active metabolites of oxygen: superoxide radical anion $\left(\mathrm{O}_{2} \overrightarrow{ }\right)$, hydrogen peroxide $\left(\mathrm{H}_{2} \mathrm{O}_{2}\right)$ and nitric oxide ( $\left.\mathrm{NO}\right)$ etc. Some of them play an important role as signaling secondary messengers, others lack specificity or reversibility required for signaling (Forman et al., 2010). Low molecular weight effector metabolites, such as cysteine, are involved in redox processes without the use of oxygen (de Beus et al., 2004; Auclair et al., 2013).

4. The main endogenous sources of ROS for redox signaling are membrane NAD(P)H oxidases (Nox1 - Nox5, Dual Oxidasel (Duox1) and Dual Oxidase2 (Duox2), specialized in ROS production, for the regu- lation of many cellular functions (Roy et al., 2015; Breitenbach et al., 2018), while Nox1, Nox2, Nox3, and Nox 5 synthesize the superoxide radical anion, and Nox4 and double oxidases DUOX1, DUOX2 predominantly produce $\mathrm{H}_{2} \mathrm{O}_{2}$ (Miyata et al., 2017; Kalyanaraman et al., 2018). In addition to them, nitric oxide synthases and the mitochondrial electron transport chain are involved in the formation of ROS (Zhao et al., 2019).

5 . Effector systems which are directly or indirectly involved in redoxregulated processes include transcription factors such as Nrf2 (nuclear factor erythroid 2-related factor 2), HIF1 $\alpha$ (hypoxia-inducible factor 1-alpha); NFkB (nuclear factor NF-kappa-B p100 subunit), MAPK (mitogenactivated protein kinase) and GTPases (Hawkins et al., 2016). Proteins Nrf2, HIF1 $\alpha, \mathrm{NF} \kappa \mathrm{B}, \mathrm{MAPK}$ are the main regulators of redox-sensitive transcriptional signaling networks in the cell. The coordinated work of these proteins initiates a transcriptional response that pleiotropically reprograms the redox homeostatic tuning of the cell, rather than simply mechanically increasing its antioxidant capacity (Hawkins et al., 2016).

An important role is also played by sirtuins - protein deacetylases, which play a unique role in redox biology due to the adjustment to $\mathrm{NAD}^{+}$ levels that can induce ROS production and regulate protein acetylation (Singh et al., 2017; Held et al., 2020), controlling such important processes such as transcription, recombination, genome stability by modifying histones, transcription factors and regulating epigenetic processes (Jing et al., 2015). 
Redox signaling is a well-regulated metabolic process in cells that controls cell growth, differentiation, proliferation and apoptosis. The interaction between the formation of oxidants and antioxidant protection is tightly regulated, maintaining redox homeostasis in cells. When regulation processes are disrupted, various pathological conditions arise, including oncology, cardiovascular and metabolic diseases (Franco et al., 2019).

Most redox reactions in biological systems are catalyzed by oxidoreductases, which indicates the presence of genetic control over these processes. A characteristic property of oxidoreductases is that the primary amino acid sequence of their apoenzyme determines the conformation of sites that is specific for such coenzymes as $\mathrm{NAD}^{+} / \mathrm{NADH}$ and $\mathrm{FAD} /$ $\mathrm{FADH}_{2}$, etc. Interaction of amino acid residues plays an important role in the spatial structure of such proteins (Cis, His and others), which determines the specificity and efficiency of intra- and intermolecular electron transport pathways (Saab-Rincon et al., 2009).

Recently, it was hypothesized that, in addition to the genetic code that allows the reproduction and determination of heredity processes, there is a redox code that identifies cellular regulatory elements and determines the principles by which biological function is activated and protected (Jones et al., 2015). The redox code includes specific reversible oxidative changes in proteins that modulate the tertiary structure of the protein, interactions, transfer, and activity, and thus bind the proteome to the redox metabolome of cells (Pinto et al., 2018).

Reactive oxygen species (ROS), formed in eukaryotic cells through aerobic metabolism, have evolved as regulators of important signaling pathways (Tsekhmistrenko \& Ponomarenko, 2013; Perillo et al., 2020), are highly reactive, and are formed as by-products during metabolic processes in various subcellular compartments: mitochondria, endoplasmic reticulum, lysosomes and peroxisomes (Di Meo et al., 2016; Snezhkina et al., 2019). ROS play an important role as intra- and extracellular messengers, encoding the functional / metabolic state of the cell for the adaptive regulation of numerous signaling pathways involved in proliferation, survival, apoptosis, and immune response (Babu \& Tay, 2019). ROS are highly reactive oxygen-containing molecules: hydroxyl $\left(\mathrm{HO}^{*}\right)$ and superoxide $\left(\mathrm{O}_{2}^{*}\right)$ free radicals and non-radical molecules, for example, hydrogen peroxide $\left(\mathrm{H}_{2} \mathrm{O}_{2}\right)$, which is less reactive than most ROS, which is a more stable and diffusible form of ROS, capable of reaching any cell compartment. $\mathrm{H}_{2} \mathrm{O}$ reacts selectively to cysteine residues in proteins and, in a low nanomolar range, can control cell signaling (Tsekhmistrenko \& Polishchuk, 2010; Marengo et al., 2016; Perillo et al., 2020).

Superoxide radical anion and hydrogen peroxide are formed during enzymatic reactions and are involved in both reversible biochemical processes of signal transmission and pathological processes associated with oxidative stress. In contrast, other ROS such as peroxynitrite $\left(\mathrm{OONO}^{-}\right)$and hydroxyl radical $\left(\mathrm{HO}^{\circ}\right)$ are not considered signaling molecules due to their high reactivity and irreversible modifications, but actively contribute to the manifestations of oxidative stress and cell damage (Brown et al., 2015; Pradedova et al., 2017). Deficiency of ROS, which are involved in signaling pathways, is associated with immune disorders, inflammation, and a decrease in the proliferative response, in part due to impaired cell signaling (Yang et al., 2013).

Recently, there have been reports that nanoparticles of metals and non-metals, products of modern nanotechnology, play a significant role in the regulation mechanisms of redox processes in the cell (SadowskaBartosz \& Bartosz, 2018; Zheng et al., 2019; Shcherbakov et al., 2020).

Nanotechnology is an interdisciplinary field of research that is based on new concepts and fundamental research and includes the synthesis, characterization and application of nanomaterials (Tsekhmistrenko et al., $2020 \mathrm{c}$ ). It is one of the fastest growing areas that have received significant application in various fields over the past ten years (Bityutsky et al., 2017; Marchiol et al., 2018; Israel et al., 2019; Xia et al., 2019). Nanomaterials are nanoobjects with a size range from 1 to $100 \mathrm{~nm}$, in at least one dimension, which have specific properties in terms of size, shape, porosity, etc. (Marchiol et al., 2018).

One of the important elements that play a significant role in the regulation of redox processes in the cell is the metalloid selenium (Se). Selenium in compounds has different oxidation states $(-2,+2,+4$ and +6$)$, which allows it to exhibit specific biological properties in systems with broad integrative functions. According to modern concepts, taking into account the achievements of "omix" technologies (Misra et al., 2019), selenium is a component of the redox interface through which the body interacts with environmental factors (exposome) and reacts in accordance with them, maintaining homeostasis at the epigenome level, genome and metabolome (Fernandes et al., 2018). Selenium is presented in two forms in eukaryotic proteins as the rare amino acids selenocysteine (SeCys) and selenomethionine (SeMet).

The term selenoprotein is used exclusively for proteins containing SeCys residues, as it is the main biologically active form of selenium in proteins. The amount of selenoproteins (selenoproteomes) may differ in different species of living organisms (Lobanov et al., 2009). Glutathione peroxidases and thioredoxin reductases are the most studied selenoproteins (Pacitti et al., 2014). They are an indispensable component of the cellular glutathione and thioredoxin systems and, therefore, are important regulators of the intracellular redox environment (Betancor et al., 2016).

Currently, selenium and its various forms have not lost their importance, but are gaining relevance in connection with the emergence of new evidence that various forms of selenium can act as antioxidant, anticancer, antifungal, antiviral, and antibacterial agents (Tsekhmistrenko et al., 2020b). The article just published shows the relationship between selenium status in humans and the course of the COVID-19 process in China (Zhang et al., 2020), which has selenium-deficient areas stretching from northeast to southwest (Rayman, 2012). Previous studies have shown that selenium deficiency in the body increases the virulence of RNA viruses such as Coxsackie B3 and influenza A (Beck et al., 2004; Hoffmann \& Berry, 2008).

Selenium deficiency in humans has caused a cardiomyopathy known as Keshan disease (an area in northeastern China where it was endemic). Seasonal fluctuations in the disease suggested the presence of a viral cofactor, which was later identified as Coxsackie B3 virus (Hoffmann \& Berry, 2008). Selenium supplementation has dramatically reduced the incidence of Keshan's disease (Hoffmann \& Berry, 2008; Rayman, 2012). Passing through a selenium-deficient animal that could not produce enough antioxidant selenoproteins to defend itself resulted in the virus mutating into a virulent form, causing more severe pathology (Beck et al., 2004; Hoffmann \& Berry, 2008).

A retrospective analysis compiled data on the selenium status of the population in China (Zhang et al., 2020) relating to the concentration of selenium in the hair, which is closely related to selenium consumption in various regions of China ( $\mathrm{Li}$ et al., 2014). A link has been established between reported cure rates for COVID-19 and selenium status. These findings are consistent with previous studies of the antiviral effects of selenium (Hou, 1997; Hoffmann \& Berry, 2008; Steinbrenne et al., 2015; Guillin et al., 2019). Multiple cellular and viral mechanisms, including selenium and selenoproteins, can influence viral pathogenicity, including virus-encoded selenium-dependent glutathione peroxidases (Guillin et al., 2019; Zhao et al., 2000). Such viral mechanisms may contribute to oxidative stress associated with many RNA viral infections (Zhao et al., 2000; Hoffmann \& Berry, 2008; Steinbrenne et al., 2015; Guillin et al., 2019); activation of viral replication (hence increased mutation frequency); and the observed higher pathogenicity or mortality in selenium deficiency as reported for SARS-CoV-2. Significant clinical benefits of selenium supplementation have also been demonstrated in other viral infections, as discussed previously (Hou, 1997; Steinbrenne et al., 2015), including HIV-1, which found a negative correlation between selenium status and mortality (Rayman, 2012). Selenium appears to be related to a number of evolutionarily different viruses due to its potential immunomodulatory effects, which are fully consistent with the many essential roles of selenium in the immune system (Hoffmann \& Berry, 2008) and its ability to influence viral mutations and evolution (Beck et al., 2004).

Thus, there is a need for further research in the context of the relationship between selenium status and disease outcome found in other viruses (Hou, 1997; Beck et al., 2004; Steinbrenne et al., 2015).

Given the fact that many RNA viral infections are accompanied by severe oxidative stress (Zhao et al., 2000; Hoffmann \& Berry, 2008, Steinbrenne et al., 2015; Guillin et al., 2019), the importance of research in the direction of the search inhibitors of oxidative stress, including various forms of selenium becomes clear.

A fundamental pathway in maintaining cellular redox homeostasis is the Keap1 / Nrf2 / ARE redox signaling system (Kelch-like ECH-associa- 
ted-protein 1/NF-E2-related-factor-2)/antioxidant response elements), which plays a key role in maintaining cellular homeostasis under stressful, inflammatory, carcinogenic and proapoptotic conditions (Taguchi et al., 2017; Kosmachevskaya et al., 2019; Tu et al., 2019; Kim et al., 2020). Recent studies show the importance of Nrf2 activity in the formation of cell metabolome and mitochondrial function (Kaidery et al., 2019; Kim et al., 2019).

Recently, there have been reports that biogenic selenium nanoparticles, obtained by green chemistry with the participation of bacteria, affect the redox-sensitive transcription factor Nrf2 (Keap1/Nrf2/ARE signaling), which activates the transcription and synthesis of a number of antioxidant and detoxifying enzymes (Song et al., 2017; Xiao et al., 2019; Xu et al., 2019; Qiao et al., 2020).

It is important to consider the regulatory mechanisms of redox processes, the structure of the repressor protein Keap1, the transcription factor $\mathrm{Nrf}$, the interaction of biogenic selenium nanoparticles with the cell with the participation of the Keap1/Nrf2/ARE signaling pathway, as a redoxsensitive signaling system of cells.

\section{Oxidative stress, nanoparticles and the Keap1/Nrf2/ARE signaling pathway}

Integration of the metabolic status of a cell with regulatory pathways is necessary to maintain cellular homeostasis (Tu et al., 2019; Zheng et al., 2019). An important element of the Keap1/Nrf2/ARE signaling system, a "sensory" protein that collects specific metabolic information and converts it into an appropriate adaptive response, is Keap1, whose reactive cysteine residues act as an electrophilic sensor tuned to respond to reactive particles originating from various endogenous and xenobiotic molecules (Taguchi et al., 2017; Kosmachevskaya et al., 2019).

As a result of changes in the intracellular redox balance, a noncovalent or covalent modification of Keap1 is observed, which is inactivated by modification of cysteine residues and loses its ability to interact with Nrf2. The nuclear transcription factor Nrf2 accumulates in the cytoplasm, which then translocates into the cell nucleus and initiates the tran- scription of antioxidant and cytoprotective genes at the antioxidant response element (ARE) loci (Bollong et al., 2018).

The Nrf2 and Keap1 proteins are linked and act as components of a single redox-sensitive signaling system (Taguchi et al., 2017; Kosmachevskaya et al., 2019). Nrf2 activity is regulated at various levels, including protein stability, transcription, and post-transcription (Tonelli et al., 2018). The Nrf2/Keapl system is present not only in mammals, but also in fish, which suggests that its role in cell protection persists throughout evolution in vertebrates (Kobayashi \& Yamamoto 2005).

\section{Nrf2 structure}

Nuclear factor (erythroid-derived 2)-like 2 (Nrf2) is a transcription factor that activates many genes encoding drug transporters, antioxidant enzymes, and phase II detoxifying enzymes such as heme oxygenase (HO-1), NAD (P) H-quinone oxidoreductase-1 (NQO-1), glutamate cysteine ligase (GCL), glutathione S-transferase (GST), catalase, superoxide dismutase (Wang et al,. 2008; Villeneuve et al., 2010). Nrf2 is a member of the Cap'n'Collar transcription factor subfamily in vertebrates, the main transcription factors for leucine zipper (bZip) (Li et al., 2019).

Under homeostasis conditions, Nrf2 has a half-life of about 20-30 minutes, undergoing constant ubiquitin-dependent protein degradation (Katoh et al., 2005). Constitutive degradation of Nrf2 allows only basal expression of its target stress response genes. However, under conditions of oxidative stress or in the presence of electrophilic xenobiotics, the activity of Keap1 decreases and Nrf2 can accumulate in the nucleus, where it activates the inducibly high expression of target genes (Canning et al., 2015).

The main control of Nrf2 stability is carried out using Kelch-like ECH-associated protein 1 (Keap1), which is a substrate adapter protein for the Cullin3 (CUL3)-RBX1 E3 ubiquitin ligase complex (Tian et al., 2018; Robledinos-Antón et al., 2019).

Studies have identified seven highly conserved domains in the structure of Nrf2, called the domains of homology NHF2-ECH (NEH) from Neh1 to Neh7 (Fig. 1), which are involved in the functions of Nrf2 (Panieri etal., 2019).

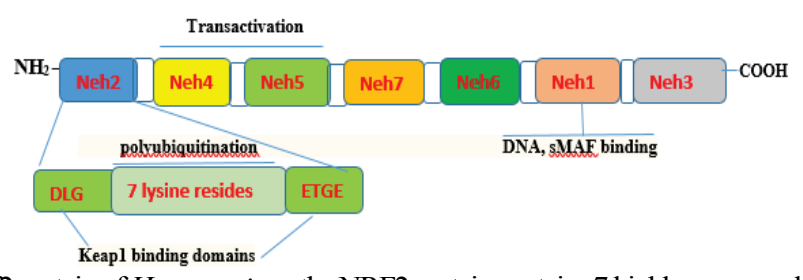

Fig. 1. Domain structure of the Nrf2 protein of Homo sapiens: the NRF2 protein contains 7 highly conserved regions (Neh). Neh1 is responsible for dimerization with the sMaf protein and binding of the ARE sequence to DNA; the Neh2 domain regulates interaction with Keap1 through the DLG and ETGE motifs, the region with 7 lysine residues is involved in polyubiquitination; domains Neh3, Neh4 and Neh5 are transactivation domains; the Neh6 domain negatively regulates the stability of NRF2; the Neh7 domain interacts with RXR $\alpha$. Keap1, Kelch-like ECH-associated protein 1; Neh, Nrf2-ECH homology; Nrf2, nuclear factor E2-related factor 2; RXR $\alpha$, retinoid X receptor $\alpha$

The N-terminal domain of Neh2 contains seven lysine residues (targets for ubiquitylation) and is a binding site for the inhibitory Keap1 protein with the formation of the Keap1 homodimer (Stefanson et al., 2014). The domain has a high-affinity motif-binding site (ETGE) and a lowaffinity motif-binding site (DLG) (Robledinos-Antón et al., 2019). Domains Neh4 and Neh5 are transcription activation domains. The Neh6 domain contains a serine-rich region that is involved in the degradation of Nrf2 (Jung et al., 2018). The Neh7 domain interacts with the retinoic Xreceptor $\alpha$, thus repressing Nrf2 (Bai et al., 2016). The Neh1 domain is responsible for DNA binding and dimerization with small sMaf proteins (musculoaponeurotic fibrosarcoma oncogene). At the C-terminus, Nrf2 contains the main domain of leucine zipper (bZip), Neh3, which is involved in the formation of heterodimers with other proteins. These heterodimers regulate genes which are involved in multiple homeostatic functions, including regulation of inflammation, redox metabolism, and proteostasis (de la Vega et al., 2016; Pajares et al., 2016; Pajares et al., 2017).

\section{Keap1 structure}

The Keap1 protein was first discovered as a necessary component for complex formation with Nrf2 and named Keap1 (Kelch-like ECH-asso- ciated protein 1) as having a structural similarity to the Drosophila Kelch protein (Itoh et al., 1999). Keap1 is a 624 amino acid homodimeric protein that functions as an adapter for the Cul3-Rbx E3 ubiquitin ligase complex (Cullinan et al., 2004; Tu et al., 2019).

Keap1 functions as a critical sensor for cellular stress. The study of its primary structure showed that Keap 1 contains 25 and 27 cysteine residues in murine and human homologues, respectively, which are distributed throughout the Keapl protein and are "sensors" for oxidation or covalent modification by electrophiles. An important fact is that 10 of these cysteines are adjacent to positively charged amino acids; this arrangement decreases the pKa of the adjacent cysteine sulfhydryl group and thus stabilizes the thiolate anion, keeping the cysteine reactive (Snyder et al., 1981). Modifications of these cysteine sulfhydryl groups, especially Cys151, Cys273 and Cys288 of the Keap1 protein, cause Nrf2 to dissociate from Keap1. Cys 273 or Cys288 are required for Keap1 repression of Nrf2 under basal conditions, and Cys151 is important for Nrf2 activation in response to electrophilic stress.

Interestingly, different reactive chemicals target different cysteines in Keap1, which contributed to the development of the "cysteine code" concept (Kansanen et al., 2013). Two residues, Cys273 and Cys288, are required by Keap1 to control Nrf2 both under basal conditions and under 
stress, while Cys151 is primarily required under stress conditions. Other Keap1 residues, including Cys226, Cys434, and Cys613, appear to be important for the perception of specific toxins (Canning et al., 2015).

Within the concept of the cysteine redox code, endogenous production of reactive oxygen species (ROS) is a highly regulated process that serves to transmit signals and can lead to modification of sensory cysteine redox "switches". The cysteine code hypothesis suggests that structurally different activators of the transcription factor Nrf2 act on different cysteines of the Keap1 protein, since cysteine modifications play a decisive role in promoting Nrf2 activation (Dinkova-Kostova et al., 2017). Thus, the Keap1-Nrf2 system is considered mainly a thiol-based sensory-effector apparatus for exogenous and endogenous electrophiles and oxidants and maintenance of redox homeostasis of cells (Yamamoto et al., 2018; Li et al., 2019). Modifications of the Keapl cysteine lead to the accumulation of Nrf2, translocation into the nucleus, and transcriptional upregulation of Nrf2-dependent cytoprotective genes (Dinkova-Kostova et al., 2017).

Keap1 consists of 5 domains (Fig. 2). The NTR (amino terminal region) is followed by BTB, which is required for Keap1 homodimerization and interaction with Cullin3 and contains a redox-sensitive cysteine residue (Cys151). In addition, cysteine residues 77 and 171 are contained, the role of which has not yet been clarified. The BTB domain is named after a broad complex of proteins from Drosophila, Tramtrack, and Bric Brac. The Drosophila bricàbrac protein and transcriptional regulators encoded by tramtrack and Broad-Complex contain a highly conserved domain of about 115 amino acids, which is called the BTB domain (Zollman et al., 1994).

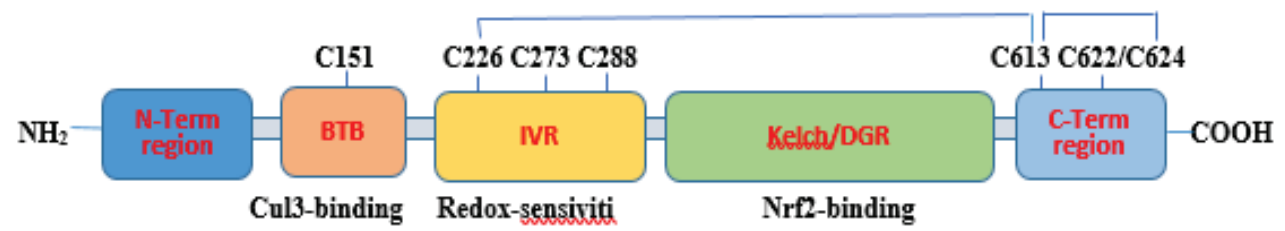

Fig. 2. The domain structure of the Homo sapiens KEAP1 protein is a 5-domain protein. NH2 (N-Term region) contains 4 cysteine residues, BTB-domain contains 3 cysteine residues, of which Cys-151 is sensory; IVR-domain - 9 cysteines, of which Cys-226, Cys-273 and Cys-288 are sensory; Kelch / DGR-domain -8 cysteines, C-Term region contains 3 sensory cysteine residues, which together with Cys- 226 act as a sensor for $\mathrm{H}_{2} \mathrm{O}_{2}$; the BTB domain is critical for KEAP1 dimerization and Cul3-based E3 ligase attachment. The IVR domain has hypercritical cysteine residues, Cys273 and Cys288, which are required to control NRF2 activity; the Kelch / DGR domain negatively regulates NRF2 activation by interacting with the conserved carboxyl terminus of the Neh2 domain; the Keap1 dimer binds one Nrf2 molecule via ETGE and DLG motifs and binds to Cullin3, which binds a RING protein such as Rbx1 to the C-terminal domain; BTB, Broad complex/Tramtrack/bric-a-brac; CTR, carboxy-terminal; DGR, double glycine repeats; IVR, intervening region; KEAP1, Kelch-like ECH-associated protein 1; Neh, NRF2-ECH homology; NFE2, nuclear factor erythroid 2; NRF2, NFE2-related factor 2; NTR, N-terminal region; RXRa, retinoid X receptor $a$

The next intermediate domain (IVR), is a cysteine-rich motif that is particularly sensitive to redox changes and influences Keapl function. The IVR domain containing 9 cysteine residues, in addition to its interaction with the $\mathrm{Cul} 3$ protein, which contains the $\mathrm{E} 3$ ligase complex together with Rbx1 (RING-box protein 1), has a coding sequence important for Keap1 localization in the cytoplasm (Furukawa et al., 2005). Sensory cysteine residues for sensitive electrophiles have been identified in the BTB (Cys151) and IVR domains (Cys273/288) (Itoh et al., 1999; Tu et al., 2019). The DGR domain (DGR double glycine repeat) contains six Kelch motifs that facilitate protein-protein interactions with Keap1 regulators, including Nrf2 and other functional partners. The CTR domain (carboxyterminal region) is important for the interaction between Keap1 and Nrf2.

\section{Keap1/Nrf2/ARE signaling pathway}

The integration of adaptive responses to various stressors plays a fundamental role in protecting cells from damage. These highly coordinated strategies aim to counteract internal disturbances, restore cellular homeostasis, and maintain cellular survival. The Nrf2/Keapl/ARE signaling pathway is a central junction of many pathways involved in cellular defense and adaptation (Kopacz et al., 2020).

By controlling the transactivation of more than 500 cytoprotective genes, the Nrf2 transcription factor is involved in the physiopathology of a number of human diseases, including cancer. In this regard, accumulating data indicate that Nrf2 may act ambivalently and may mediate tumour suppressive or pro-oncogenic functions, depending on the specific biological context of its activation. Thus, a better understanding of the mechanisms governing Nrf2 functions and the most appropriate context for its activation is a prerequisite for the development of effective therapeutic strategies based on Nrf2 modulation (Panieri et al., 2020).

Nrf2 activity is tightly controlled and regulated in mammalian cells. Under normal physiological conditions, Nrf2 functioning is maintained at a low level, since Nrf2 is continuously degraded in the ubiquitin proteasome system. There are many ubiquitin ligase systems that are responsible for the degradation of $\mathrm{Nrf} 2$ in the proteasome system. One of such complex ligase systems is the Cullin 3 (Cul3) RING-box 1 (RBX1) E3 ubiquitin ligase system. Keap1 acts as a substrate for the ubiquitination of the Nrf2 protein (Cullinan et al., 2004; Kobayashi et al., 2004; Zhang et al., 2004) and is located in the cytoplasm (Shah et al., 2018).
Constitutive activation of $\mathrm{Nrf} 2$ allows only minor expression of its target stress response genes as part of steady state function. However, under conditions of oxidative stress or in the presence of electrophilic xenobiotics, the Keap1/Nrf2 complex dissociates, $\mathrm{Nrf} 2$ is translocated into the nucleus, where it activates an inducibly high expression of target genes. Thus, Keapl functions as a critical sensor for cellular stress. Its high redox sensitivity is determined by the number of cysteine residues that are distributed throughout the Keap1 protein and are sensory targets of oxidation or covalent modification by electrophiles or ROS.

Under stationary conditions, the Keap1 homodimer binds to one Nrf2 molecule through its domains DLG and ETGE, and Nrf2 has a short halflife of about 10-30 min; therefore, Keap1-promotes high proteolysis of Nrf2 and maintains extremely low basal Nrf2 levels (Nguyen et al., 2003). As a result of oxidative stress, Keapl is oxidized in reactive cysteine residues, which leads to inactivation of Keap1, stabilization of Nrf2, and translocation into the nucleus. The DGR regions in Keap1 are used to recognize two primary sequences of motifs ETGE and DLG in the Neh2 domain of the Nrf2 protein (Padmanabhan et al., 2008). The interactions between Nrf2 and Keap1 are as follows: Keap1 molecules can dimerize with each other using the BTB domain to interact with Cullin-3, with two Keap1 proteins binding to one $\mathrm{Nrf2}$ protein in a 2: 1 ratio (Tong et al., 2006), while the overlapping ETGE and DLG motifs in Nrf2 bind to two Keap1 proteins with different affinities: one Keap1 strongly binds to ETGE $\left(\mathrm{K}_{\mathrm{a}}=\right.$ $2 \times 10^{8} \mathrm{M}^{-1}$ ), the Nrf2 motif, and the other Keapl interacts with the DLG motif with weak affinity $\left(\mathrm{K}_{\mathrm{a}}=1 \times 10^{6} \mathrm{M}^{-1}\right)$ (Tong et al., 2007). Based on these data, the so-called "hinge and latch" hypothesis was formulated to elucidate the mechanism of Nrf2 regulation by Keap1, in which ETGE ("loop") regulates the interaction with high affinity between the ETGE motif Nrf2 and Keap1. This interaction is not influenced by inducers of oxidative stress, and the latch is involved in the displacement of the DLG Nrf2 motif from Keap1 in response to the action of Nrf2 inductors (Uruno et al., 2011). According to this model, the DLG motif ("latch") cyclically switches between closed (interaction of two sites) and open (ETGE only) conformation (McMahon et al., 2006; Baird et al., 2013). In a closed conformation with Keap1, Nrf2 is ubiquitylated and degraded by the proteasome (Fig. 4) (Zhang et al., 2004; Tong et al., 2006). Keap1 works as a dimeric redox substrate for Cullin-based ubiquitin ligase E3, which inhibits the transcriptional activity of $\mathrm{Nrf} 2$ through ubiquitination and proteasome degradation. This signaling is known as the canonical pathway 
(Staurengo-Ferrari et al., 2018). Under basal (canonical) conditions, Nrf2 binds to Keap1 and undergoes rapid degradation under the action of the ubiquitin-proteasome system. A small protein called ubiquitin plays a vital role in virtually every aspect of cell life. Among the various signaling outcomes associated with ubiquitination, the most well-established is the targeted degradation of substrates through the proteasome. Cells need mechanisms to help break down proteins that are not needed at the moment or have lost their functionality. Eukaryotic cells possess two main control pathways that maintain proteome homeostasis - the autophagy-lysosome system and the ubiquitin-proteasome (UPS). UPS are responsible for the degradation of short-lived regulatory proteins or misfolded proteins with the participation of the $26 \mathrm{~S}$ proteasome. The proteasome, the most complex protease known, cleaves proteins that have been conjugated to ubiquitin.

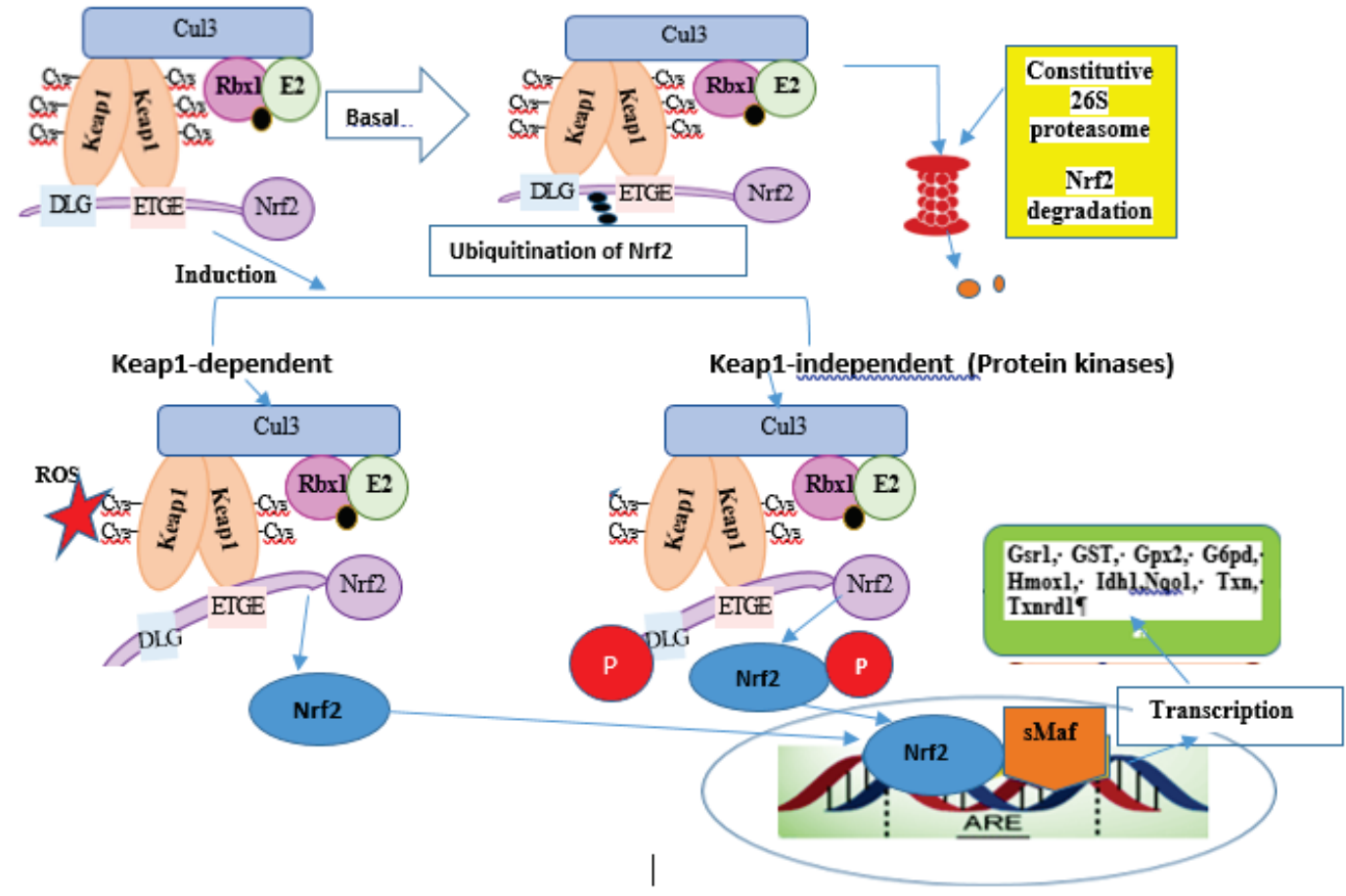

Fig. 3. Keap1-dependent and Keap1-independent signaling pathway for Nrf2 activation: under homeostatic conditions, Nrf2 is localized in the cytoplasm due to its association with the Keap1-Cul3-Rbx1 complex, which leads to continuous ubiquitination of Nrf2 and its proteasome degradation; 1 - Keap1-dependent activation pathway; in response to oxidative stress, Nrf2 dissociates from Keap1 (mainly as a result of oxidation of cysteine residues in Keap1), translocates into the nucleus, and heterodimerizes with one of the small proteins Maf; this complex activates ARE-dependent gene expression of a number of antioxidant and cytoprotective proteins; 2 - the Keap1-independent pathway of Nrf2 activation occurs with the participation of signaling cascades of protein kinases that phosphorylate Nrf2, causing it to be cleaved from the Keap1 repressor, followed by translocation into the nucleus and activation of the corresponding genes

Proteasome adaptability, which is unique among proteases, allows cells to control the ubiquitin-proteasome pathway globally (Finley et al., 2012; Bard et al., 2018; Samant et al., 2018; Finley \& Prado, 2020). Proteasome $26 \mathrm{~S}$ is the main protease in eukaryotic cells responsible for protein degradation both in the cytosol and in the nucleus. The attachment of ubiquitin targets the wasted proteins to the proteasome. Modification by ubiquitin, covalently attached to the protein side chains of lysine with the participation of many ubiquitin ligases and conjugating enzymes, enters the proteosome, where the substrate is degraded (Bard et al., 2018). Degradation of the vast majority $(80-90 \%)$ of intracellular proteins is carried out by the $26 \mathrm{~S}$ proteasome. After induction, cysteine residues in Keap1 are modified, the activity of ubiquitin ligase E3 is suppressed, and Nrf2 levels are increased. Activated Nrf2 enters the nucleus and dimerizes with MAF to facilitate the transcription of ARE-dependent genes (Panieri et al., 2020).

Reactive oxygen species or electrophilic reaction with certain cysteine residues cause conformational changes in Keap1 and prevent proteasomal degradation of $\mathrm{Nrf} 2$. The cysteine-enriched Keap1 protein regulates active Nrf2 degradation under basal conditions by acting as an adapter to cullin3 (Cul3) ringbox1 ( $\mathrm{Rbx} 1)$ containing the $\mathrm{E} 3$ ubiquitin ligase complex.

The exact mechanism by which cysteine modifications in Keapl lead to Nrf2 activation is unknown, but there are two prevailing, but not mutually exclusive, models. The first is a "loop and latch" model, in which modifications of Keap1 in thiol residues found in IVR Keapl can disrupt interaction with $\mathrm{Nrf2}$, causing displacement of lysine residues in Nrf2, which can no longer be polyubiquitinylated. In the second model the thiol modification causes the dissociation of Cul3 from Keap1.

In both models, Keap1, modified by an inducer and bound to Nrf2, is inactivated, and therefore, newly synthesized Nrf2 proteins bypass Keap1 and are translocated into the nucleus, bind to ARE, and control the expression of $\mathrm{Nrf} 2$ target genes such as $\mathrm{NAD}(\mathrm{P}) \mathrm{H}$ quinone oxidoreductase 1 (NQO1), heme oxygenase 1 (HMOX1), glutamate cysteine ligase (GCL) and glutathione S-transferase (GST). In addition to modifications of Keap1 thiols leading to the induction of the target gene $\mathrm{Nrf}$, proteins such as p21 and p62 can bind to Nrf2 or Keap1, thereby disrupting the interaction between Nrf2 and Keap1 (Kansanen et al., 2013). As a result of the modification of Keapl cysteine residues, the protein changes its conformation and loses its affinity for the low affinity DLG motif, the "latch" slips off, and Nrf2 hangs on the "loop" - the high affinity ETGE motif. This leads to impaired ubiquitination of $\mathrm{Nrf} 2$ and, consequently, its proteasome degradation (but not association with Keap1), the pool of inhibitor molecules is saturated and the content of free Nrf2 increases, and the time of its existence in cells increases to 100-200 min (Baird et al., 2011).

Cysteine residues in Keap1 determine reactive oxygen species (ROS) or electrophiles in the cellular environment, causing conformational changes in Keap1. The modified Keapl can disrupt its interaction with the low affinity DLG motif, while the high affinity ETGE motif remains associated with Keap1. Since the DLG motif does not bind to Keap1, it affects the orientation of lysine residues in the Neh2 domain of Nrf2, preventing its ubiquitination and degradation. After the restoration of redox homeostasis, Keap1 moves into the nucleus and controls the nuclear export of $\mathrm{Nrf2}$ for subsequent proteasomal degradation in the cytoplasm.

To avoid degradation and activate the transcription of ARE-driven genes, the conformation of the Nrf2 - Keapl complex must change. This occurs when Keap1 is covalently modified in its sensitive cysteine residues, which affects its conformation and stabilizes $\mathrm{Nrf2}$ in a non-degradable complex (Dinkova-Kostovaetal.,2002; Zhangetal.,2003; Baird et al., 2013). 
In addition, while controlled canonical activation of $\mathrm{Nrf} 2$ is required for cancer chemoprophylaxis, uncontrolled or prolonged non-canonical activation of Nrf2 promotes the development of cancer and chemoresistance, acting as a double-edged sword capable of mediating tumour suppressive or pro-cancer functions, depending on the specific biological context of its activation (Panieri et al., 2020a).

Therefore, understanding the mechanisms that regulate the Keapl/ Nrf2/ARE signaling pathway and the most suitable inducers of its activation is a prerequisite for the development of effective therapeutic strategies and the development of methods for the treatment or prevention of diseases (de la Vega et al., 2016; Tian et al., 2018; Panieri et al., 2020a).

\section{Nanoparticles as inducers (activators and inhibitors) of the Keap1/Nrf2/ARE signaling pathway}

Many Nrf2 activators are electrophilic molecules that covalently modify the cysteine residues of the Keap1 protein (Robledinos-Antón et al., 2019). Research shows that Nrf2 inducing agents such as triterpenoids, diethyl maleate, dimethyl fumarate, sulforaphane, curcumin, tert-butylhydroquinone modify the Cys151 residue to induce Nrf2, while 2-cyano3,12-dioxooleana-1,9-diene-28-imidazolide, cadmium chloride and arsenic activate Nrf2 independently of Cys151 (Robledinos-Antón et al., 2019). Differential reactivity of cysteines in Keap1, "cysteine code", however, does not explain how this leads to different toxin-dependent activation genes with the transcription factor Nrf2 (Mathew \& Hammarsten, 2016). Nanoparticles can serve as Keap1/Nrf2 inductors, as demonstrated in various studies (Song et al., 2017; Xiao et al., 2019).

For the synthesis of nanoparticles by traditional physical and chemical methods, it is necessary to use reducing agents, which can have a toxic effect when used by humans, they are environmentally unacceptable and expensive (Shah et al., 2019). Large-scale synthesis also faces many challenges, such as low stability and less monodispersity (Manoj et al., 2018).

The natural "biolaboratory", consisting of plants, algae, fungi, yeast, bacteria and various biomolecules in their composition, plays an active role in the formation of nanoparticles of various shapes, sizes and properties, and is the driving force for the development of safer and more ecological methods for the synthesis of nanoparticles by these bionanofactories (Sharma et al., 2019; Tsekhmistrenko et al., 2020).

The introduction of the concepts of "green chemistry" and "nanotechnology" is one of the revolutionary events in science that influenced the contribution to research on environmental safety and reduction of the size of objects. The combination of these two fields paved the way for a new "green" and nanoscale oriented science called "green nanotechnology" or bionanotechnology (Tsekhmistrenko et al., 2020).

Bacterial green synthesis is especially important for the production of nanoparticles because of their diversity, high adaptability to extreme conditions (Wang et al., 2018), low energy consumption and regulation of biogenic synthesis processes (Fang et al., 2019; Tsekhmistrenko et al., 2020).

Thanks to the "greening" of the processes of synthesis of nanoparticles using biological systems and their components, there is a decrease in the load on the environment and an increase in economic efficiency, which opens up additional opportunities for the creation of nanoparticles with a given composition and properties. Each nanosystem is unique and specific in its formulation, compatibility with active molecules, choice of excipients and kinetics, as well as biological effectiveness (Tsekhmistrenko et al., 2018; Dhapte \& Pokharkar, 2019; Tsekhmistrenko et al., 2020a).

At present, much attention is drawn to selenium nanoparticles (SeNP) obtained by the methods of "green" synthesis. Synthesized by the methods of bionanotechnology SeNP has many applications in the field of medical and pharmaceutical research. Selenium nanoparticles having oncosuppressive, antioxidant and antimicrobial activity are used in biology, medicine, agriculture (Bityutskyy et al., 2019; Tsekhmistrenko et al., 2019, 2020a; Akçay \& Avc1, 2020; Kumar et al., 2020).

Song et al. (2017) synthesized biogenic nanoselenium nanoparticles (BNSe) with the participation of Enterobacter cloacae Z0206 bacteria, with the help of which sodium selenite was transformed into spherical selenium nanoparticles with a stabilizing protein shell, the particle size of which varied from 80 to $250 \mathrm{~nm}$ (on average $139.4 \pm 7.4 \mathrm{~nm}$ ). BNSe particles were synthesized intracellularly and secreted from the cell.
The experiments compared the efficiency of biogenic selenium nanoparticles with the organic form of selenium (selenomethionine) and selenium nanoparticles obtained by physicochemical methods (most NPSe particles had a size ranging from 40 to $600 \mathrm{~nm}$ (mean value $121.0 \pm 10.3 \mathrm{~nm}$ ).

It is known that the classical definition of nanoparticles assumes their diameter is from 1 to $100 \mathrm{~nm}$, but the unique physicochemical (size, shape, chemical composition, stability) and biological properties of these nanoparticles synthesized by bionanotechnology methods ("green synthesis") allow them to be classified as nanostructures. The monodispersity of biogenic SeNP and their stability within this range is due to organic material consisting of biomolecules produced by bacterial cells, which are involved in controlling the diameter of nanoparticles (Wang, 2010).

On a model of mouse cells with oxidative stress induced by diquat (a bipyridyl herbicide that promotes the formation of a superoxide radical) in the intestinal cells of mice, it was shown that BNSe particles protect the intestinal barrier and preserve the redox homeostasis of intestinal cells more efficiently than selenomethionine and nanoselenes synthesized by chemical methods (Song et al., 2017).

In vitro studies with porcine small intestine epithelial cells (IPEC-J2) have shown the following effects: BNSe particles protect the epithelial barrier from oxidative stress, reduce cell apoptosis, improve redox homeostasis, activate the transcription factor Nrf2, enhance the expression of genes for cytoprotective and antioxidant compounds, such as TXNRD-1, NADPH dehydrogenase (NQO)-1, heme oxygenase-1 (HO-1) and thioredoxin. Subsequent study of the mechanism by which BNSe activate the Nrf2-ARE pathway and affect the physiological function of intestinal epithelial cells demonstrated that treatment of IPEC-J2 cells with biogenic nanoselenium particles significantly increased phosphorylated Nrf2 levels without noticeable effect on Keapl protein levels (Xiao et al., 2019), which indicates that activation of $\mathrm{Nrf} 2$ occurs in a Keap1-independent pathway, without the involvement of its sensory cysteines.

There are various Keap1-independent pathways for Nrf2 activation, such as the activation of protein kinase signaling cascades by molecules that modify the cellular redox status (Shin et al., 2019). It has been shown that phosphorylation at a specific amino acid residue Nrf2 (Ser-40) can increase its stability and transactivating activity (Nguyen et al., 2003). Typical signaling pathways of protein kinases include phosphatidylinositol 3-kinase (PI3K), mitogen-activated protein kinase (MAPK), protein kinase C (PKC), and Glycogen synthase kinase-3 (GSK3).

Phosphorylation of Nrf2 by PI3K, PKC, c-Jun N-terminal kinase (JNK), and extracellular signal-regulated kinase (ERK) provides up-regulation, while $\mathrm{p} 38$ MAPK (MAP kinase mitogen-activated protein kinase) regulates the Nrf2 pathway both positively and negatively (Yu et al., 2000; Huang et al., 2002; Kobayashi et al., 2005; Tong et al., 2006). Another important serine / threonine-specific protein kinase (AKT) is involved in various cellular processes, including metabolism, apoptosis, as well as in several oncopathologies and cardiovascular diseases (Zhao \& Zeng, 2020).

In the above studies (Xiao et al., 2019) it was shown that BNSe particles activated the Nrf2-ARE pathway through p38, ERK 1/2, and AKTmediated phosphorylation of $\mathrm{Nrf}$, followed by an improvement in the antioxidant function of intestinal epithelial cells.

This initiates the transcription of antioxidant and detoxification enzymes, such as $\mathrm{NAD}(\mathrm{P}) \mathrm{H}:$ quinone oxidoreductase 1 (NQO1), heme oxygenase-1 (HO-1), a cytoprotective enzyme that regulates antioxidant and inflammatory reactions, thioredoxin reductase (TXNRD) and thioredoxin (Fig. 4). An important resulting factor in the activation of Nrf2 by biogenic nanoselen is the transcription and synthesis of the components of the thioredoxin system consisting of thioredoxin (Trx) and thioredoxin reductase (TXNRD). TXNRD is a selenoenzyme and NADPHdependent flavoprotein that reduces oxidized Trx to its dithiol form to maintain redox homeostasis of cells and protects DNA from damage associated with oxidative stress (Lee et al., 2019).

In studies by Xu et al. (2018) it was found that biogenic selenium nanoparticles (BNSe2) synthesized using the bacteria Lactobacillus casei ATCC 393 were $50-80 \mathrm{~nm}$ in size, concentrated in the intracellular space, and were stabilized by the protein-polysaccharide shell. It was shown that the toxicity of various forms of selenium decreased in the following order: sodium selenite $>$ selenium methionate $>$ BNSe2. It was found that BNSe2 after endocytosis induced apoptosis of HepG2 cells with the par- 
ticipation of caspases, reduced oxidative stress in intestinal epithelial cells induced by prooxidants (diquat, $\mathrm{H}_{2} \mathrm{O}_{2}$ ), decreased the concentration of the end product of lipid peroxidation (malondialdehyde), increased the activity of selenate-dependent glutase-dependent glutase. In a model of hydrogen peroxide-induced oxidative damage to epithelial cells of the human colon mucosa (NCM 460), it was demonstrated that BNSe2, synthesized by L. casei ATCC 393, reduced ROS levels, normalized mitochondrial membrane potential (MMP), and retained the permeability of these cells (Qiao et al., 2020). In addition, BNSe2 increased the levels of Nrf2, heme oxygenase-1 (HO-1), NAD(P)H dehydrogenase (quinone)-1(NQO-1) and neutralized damage to the mitochondrial ultrastructure caused by oxidative stress (Fig. 5). The addition of a low molecular weight inhibitor Nrf2 (ML385), which binds to Neh1, the Nrf2 domain and prevents the formation of the MAF-Nrf2 protein complex with regulatory DNA binding sequences, as a result of which transcription of antioxidant enzyme genes does not occur and the protective effect of biogenic nanoselen is eliminated.

Thus, it has been demonstrated that biogenic BNSe2 synthesized using L. casei ATCC 393 protects the intestinal epithelial barrier function from the consequences of oxidative damage, normalizes mitochondrial functions with the participation of the Nrf2/ARE signaling pathway.

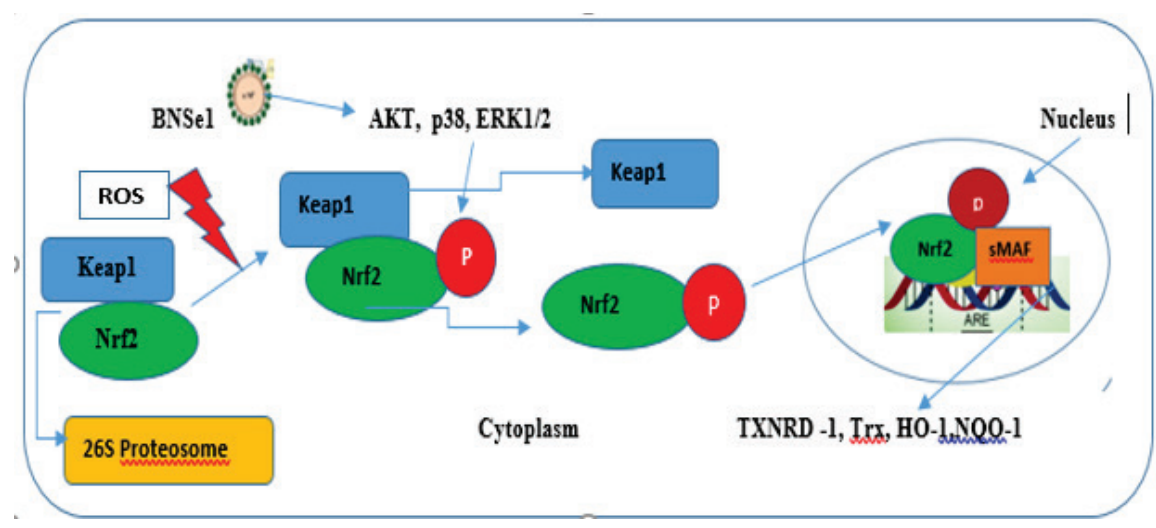

Fig. 4. Mechanisms of activation of the Nrf2/ARE pathway by biogenic nanoselenium nanoparticles (BNSe) synthesized with the participation of E. cloacae Z0206 bacteria in intestinal epithelial cells (IPEC-J2): under homeostasis conditions, Nrf2 forms a complex with Keap1 in the cytosol, which promotes polyubiquitination and $\mathrm{Nrf} 2$ degradation by the $26 \mathrm{~S}$ proteasome; under oxidative stress, treatment of cells with BNSe1 particles activates Nrf2 using the Keap1 - independent activation pathway via p38, ERK1/2, and AKT-mediated phosphorylation of Nrf2; Nrf2 is cleaved from the Keap1 repressor, translocated into the cell nucleus, binds to ARE and SMAF, which causes the expression of antioxidant and cytoprotective genes: thioredoxin

reductase-1 (TXNRD-1), NADPH-quinone (HO-1) and thioredoxin (Trx); Nrf2, Nuclear factor erythroid 2-related factor 2; Keap1, Kelch-like ECHassociated protein 1; ARE, antioxidant response element; Ub, ubiquitin; AKT-; p38-; ERK1/2-P, phosphate (adapted from Song et al., 2017; Xiao et al., 2019)

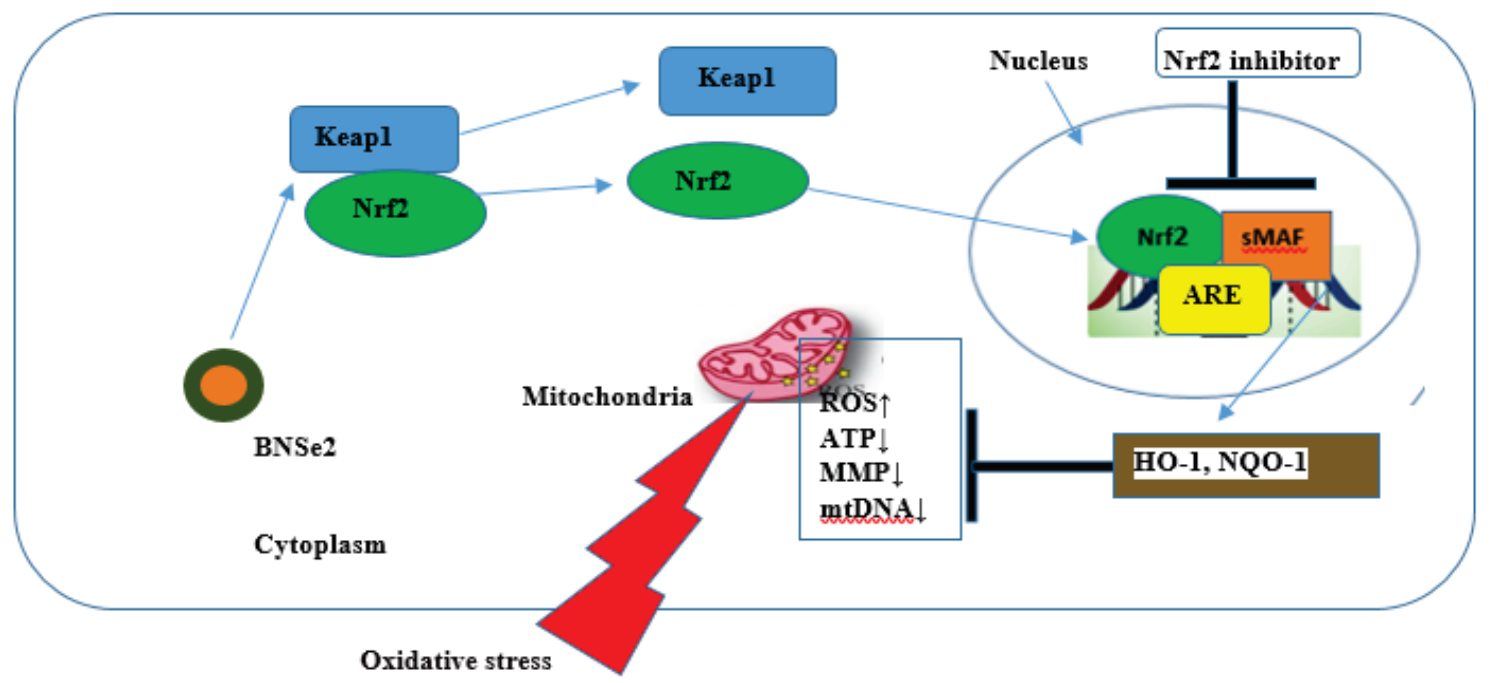

Fig. 5. The proposed mechanism of the protective action of biogenic BNSe2, synthesized by Lactobacillus casei ATCC 393, on the intestinal epithelial barrier, which neutralizes oxidative damage: the Nrf2 inhibitor eliminates the antioxidant effect of BNSe2, preventing the formation of the sMaf-Nrf2 complex and its binding to ARE. BNSe2, biogenic selenium nanoparticles; ARE, antioxidant response element; MMP, mitochondrial membrane potential (adapted from Xu et al. (2019), Qiao et al. (2020))

The dualism of mitochondria is manifested in the fact that they are the main source of reactive oxygen species (ROS) and, at the same time, a highly sensitive target of oxidative stress. Mitochondrial damage leads to bioenergetic dysfunction and cell death, both through necrosis and apoptosis. It has also been shown that activation of Nrf2 by the inducer sulforaphane activates mitochondrial antioxidants and protects mitochondria from dysfunction, as well as nerve cells from damage and death (Goodfellow et al., 2020). Interestingly, depending on the type of bacteria involved in biogenic synthesis of nanoparticles, particle size and stabilizing shell, the signaling pathway is activated via the Keap1-independent pathway of Nrf2 activation, including phosphorylation of Nrf2 by various protein kinases (Xiao et al., 2019) and the Keap1-dependent pathway. In contrast to biogenic selenium nanoparticles, nanoselenium particles obtained by chemical methods participate in antioxidant protection by including selenoproteins in the structure (Song et al., 2017).

The gastrointestinal tract is one of the key sources of ROS, therefore the stability of the intestinal barrier is critical to maintaining its healthy and stable condition. A significant role of activation of the Keap1/Nrf2/ARE signaling pathway in maintaining the intestinal barrier is as follows: the damage to the intestinal mucosa is reduced; intestinal inflammation decreases, suppressing the inflammatory pathway; intestinal permeability is normalized by acting on intestinal epithelial proteins and cell apoptosis; processes of differentiation and function of $\mathrm{T}$ cells are regulated; crossinteraction between the Keap1-Nrf2 pathway and autophagy controls oxidative stress in intestinal cells (Wen et al., 2019). Currently, alternative mechanisms of regulation of $\mathrm{Nrf} 2$ functioning have been identified, in- 
cluding phosphorylation of $\mathrm{Nrf} 2$ by various protein kinases (PKC, PI3K/Akt, GSK-3 $\beta$, JNK), interaction with other protein partners (p21, caveolin-1) and epigenetic factors (micro-RNA-144, -28 and -200a and promoter methylation). It has been established that non-covalent activation of Nrf2 provides greater cellular protection than covalent activation (Liu et al., 2019), which stimulates research in this direction.

These and other processes are potentially important determinants of Nrf2 activity and, therefore, may contribute to the maintenance of cellular homeostasis. A vital factor in the functioning of many transcription factors is their spatio-temporal regulation. It was found that Nrf2 "turns on" in response to a stimulus and "off" when the stimulus is removed. It is for this reason that this pathway is strictly regulated by a number of different mechanisms responsible for preventing excessive Nrf2 activation, which can cause various pathologies.

\section{Conclusion}

The transcription factor Nrf2 provides cellular protection by maintaining cellular redox homeostasis and proteostasis by regulating the expression of genes containing antioxidant-responding element (ARE) promoters. Under homeostatic conditions, Nrf2 levels are low; it is bound to the Keap1-Cul3-Rbx1 complex, which leads to continuous Nrf2 ubiquitination and its proteasome degradation. The repressor protein Keapl is a molecular sensor of redox balance inside the cell; it contains sensory cysteines (Cys151, Cys273, Cys288, Cys226, Cys434 and Cys613), which form discrete sensors that respond to various types of electrophiles, realizing the so-called "cysteine code". Modification of specific combinations of Keap1 cysteines by oxidation or alkylation leads to dissociation of the Keap1 - Nrf2 complex, inhibition of proteosomal degradation of Nrf2, its translocation into the nucleus, heterodimerization from MAF, and activation of ARE-dependent expression of genes for antioxidant and cytoprotective proteins. Alternative mechanisms of $\mathrm{Nrf} 2$ regulation include phosphorylation of Nrf2 by various protein kinases (PKC, PI3K/Akt, GSK-3ß, $\mathrm{JNK}$ ), interactions with other protein partners, and epigenetic factors.

The importance of NRF2 modulating compounds and their pleiotropic effects make it promising to search for and create composite materials based on natural Nrf2 activators with the active participation of nanoparticles, primarily biogenic ones, with their possible functionalization for targeting, transportation, and effective use. In recent years, inflammatory bowel diseases have increased significantly due to changes in dietary habits and the effects of various environmental factors (exposureome). Prevention and treatment of this type of disease require an understanding of the defense systems functioning mechanisms, including the activation of the transcription factor Nrf2 in organs or tissues. Among them the gastrointestinal tract, the mucous membrane of which is a critical barrier and its defects are associated with a wide range of diseases and represent a new therapeutic target. The Keap1/Nrf2 signaling pathway plays an important role in maintaining the integrity of the intestinal barrier, reducing intestinal damage and inflammation, participating in the regulation of the inflammatory pathway in various disease models, suppressing the formation of reactive oxygen species, increasing cell survival, and activating the transcription of target genes encoding a cascade of antioxidant and cytoprotective enzymes. Thus, it is clear that Nrf2-dependent gene activation enhances protection against oxidative stress. Since many diseases have an oxidative stress component, the decisive role of the Nrf2 signaling pathway has led to extensive clinical and laboratory research. Detailed knowledge of thiol-dependent and independent mechanisms of red6ox signaling under physiological and pathological conditions will lead to a deeper understanding of the role of the redox component in human and animal health and disease. Modulation of the Keap1/Nrf2/ARE signaling pathway identifies promising therapeutic targets for a variety of redoxmediated diseases.

\section{References}

Akçay, F. A., \& Avci, A. (2020). Effects of process conditions and yeast extract on the synthesis of selenium nanoparticles by a novel indigenous isolate Bacillus sp. EKT1 and characterization of nanoparticles. Archives of Microbiology, 202, 2233-2243.
Auclair, J. R., Brodkin, H. R., D’Aquino, J. A., Petsko, G. A., Ringe, D., \& Agar, J. N. (2013). Structural consequences of cysteinylation of $\mathrm{Cu} / \mathrm{Zn}$-superoxide dismutase. Biochemistry, 52(36), 6145-6150.

Babu, R. K., \& Tay, Y. (2019). The Yin-Yang regulation of reactive oxygen species and microRNAs in cancer. International Journal of Molecular Sciences, 20(21), 5335 .

Bai, X., Chen, Y., Hou, X., Huang, M., \& Jin, J. (2016). Emerging role of NRF2 in chemoresistance by regulating drug-metabolizing enzymes and efflux transporters. Drug Metabolism Reviews, 48(4), 541-567.

Baird, L., \& Dinkova-Kostova, A. T. (2011). The cytoprotective role of the Keap1Nrf2 pathway. Archives of toxicology, 85(4), 241-272.

Baird, L., Llères, D., Swift, S., \& Dinkova-Kostova, A. T. (2013). Regulatory flexibility in the Nrf2-mediated stress response is conferred by conformational cycling of the Keap1-Nrf2 protein complex. Proceedings of the National Academy of Sciences, 110(38), 15259-15264.

Bard, J. A., Goodall, E. A., Greene, E. R., Jonsson, E., Dong, K. C., \& Martin, A. (2018). Structure and function of the $26 \mathrm{~S}$ proteasome. Annual review of biochemistry, 87, 697-724.

Beck, M. A., Handy, J., \& Levander, O. A. (2004). Host nutritional status: The neglected virulence factor. Trends in Microbiology, 12(9), 417-423.

Bellezza, I., Giambanco, I., Minelli, A., \& Donato, R. (2018). Nrf2-Keap1 signaling in oxidative and reductive stress. Biochimica et Biophysica Acta - Molecular Cell Research, 1865(5), 721-733.

Betancor, M. B., Dam, T. M., Walton, J., Morken, T., Campbell, P. J., \& Tocher, D. R. (2016). Modulation of selenium tissue distribution and selenoprotein expression in Atlantic salmon (Salmo salar L.) fed diets with graded levels of plant ingredients. British Journal of Nutrition, 115(8), 1325-1338.

Bityutsky, V., Tsekhmistrenko, O., Tsekhmistrenko, S., Spyvac, M., \& Shadura, U. (2017). Perspectives of cerium nanopaticles use in agriculture. The Animal Biology, 19(3), 9-17.

Bityutskyy, V., Tsekhmistrenko, S., Tsekhmistrenko, O., Melnychenko, O., \& Kharchyshyn, V. (2019). Effects of different dietary selenium sources including probiotics mixture on growth performance, feed utilization and serum biochemical profile of quails. In: Modern development paths of agricultural production. Springer, Cham. Pp. 623-632.

Bollong, M. J., Lee, G., Coukos, J. S., Yun, H., Zambaldo, C., Chang, J. W., Chin, E. N., Ahmad, I., Chatterjee, A. K., Lairson, L. L., Schultz, P. G., \& Moellering, R. E. (2018). A metabolite-derived protein modification integrates glycolysis with KEAP1-NRF2 signalling. Nature, 562(7728), 600-604.

Breitenbach, M., Rinnerthaler, M., Weber, M., Breitenbach-Koller, H., Karl, T., Cullen, P., Basu, S., Haskova, D., \& Hasek, J. (2018). The defense and signaling role of NADPH oxidases in eukaryotic cells. Wiener Medizinische Wochenschrift, 168(11-12), 286-299.

Brown, D. I., \& Griendling, K. K. (2015). Regulation of signal transduction by reactive oxygen species in the cardiovascular system. Circulation Research, 116(3), 531-549.

Canning, P., Sorrell, F. J., \& Bullock, A. N. (2015). Structural basis of Keap1 interactions with Nrf2. Free Radical Biology and Medicine, 88, 101-107.

Cullinan, S. B., Gordan, J. D., Jin, J., Harper, J. W., \& Diehl, J. A. (2004). The Keap1-BTB protein is an adaptor that bridges Nrf2 to a Cul3-based E3 ligase: Oxidative stress sensing by a Cul3-Keap1 ligase. Molecular and Cellular Biology, 24(19), 8477-8486.

de Beus, M. D., Chung, J., \& Colón, W. (2004). Modification of cysteine 111 in $\mathrm{Cu} / \mathrm{Zn}$ superoxide dismutase results in altered spectroscopic and biophysical properties. Protein Science, 13(5), 1347-1355.

de la Vega, M. R., Dodson, M., Chapman, E., \& Zhang, D. D. (2016). NRF2-targeted therapeutics: New targets and modes of NRF2 regulation. Current Opinion in Toxicology, 1, 62-70.

Dhapte, V., \& Pokharkar, V. (2019). Nanosystems for drug delivery: Design, engineering, and applications. In: Green Synthesis, Characterization and Applications of Nanoparticles. Elsevier. Pp. 321-345.

Di Meo, S., Reed, T. T., Venditti, P., \& Victor, V. M. (2016). Role of ROS and RNS sources in physiological and pathological conditions. Oxidative Medicine and Cellular Longevity, 2016, 1245049.

Dinkova-Kostova, A. T., Holtzclaw, W. D., Cole, R. N., Itoh, K., Wakabayashi, N., Katoh, Y., Yamamoto, M., \& Talalay, P. (2002). Direct evidence that sulfhydryl groups of Keap1 are the sensors regulating induction of phase 2 enzymes that protect against carcinogens and oxidants. Proceedings of the National Academy of Sciences, 99(18), 11908-11913.

Dinkova-Kostova, A. T., Kostov, R. V., \& Canning, P. (2017). Keap1, the cysteinebased mammalian intracellular sensor for electrophiles and oxidants. Archives of Biochemistry and Biophysics, 617, 84-93.

Eggler, A. L., Gay, K. A., \& Mesecar, A. D. (2008). Molecular mechanisms of natural products in chemoprevention: Induction of cytoprotective enzymes by Nrf2. Molecular Nutrition and Food Research, 52(S1), S84-S94.

Fang, X., Wang, Y., Wang, Z., Jiang, Z., \& Dong, M. (2019). Microorganism assisted synthesized nanoparticles for catalytic applications. Energies, 12(1), 190. 
Fernandes, J., Hu, X., Smith, M. R., Go, Y. M., \& Jones, D. P. (2018). Selenium at the redox interface of the genome, metabolome and exposome. Free Radical Biology and Medicine, 127, 215-227.

Finley, D., \& Prado, M. A. (2020). The proteasome and its network: Engineering for adaptability. Cold Spring Harbor Perspectives in Biology, 12(1), a033985.

Finley, D., Ulrich, H. D., Sommer, T., \& Kaiser, P. (2012). The ubiquitin - proteasome system of Saccharomyces cerevisiae. Genetics, 192(2), 319-360.

Forman, H. J., Maiorino, M., \& Ursini, F. (2010). Signaling functions of reactive oxygen species. Biochemistry, 49(5), 835-842.

Franco, M. C., Carreras, M. C., \& Hannibal, L. (2019). Molecular basis of redox signaling. Oxidative Medicine and Cellular Longevity, 2019, 6414975-6414975.

Furukawa, M., \& Xiong, Y. (2005). BTB protein Keap1 targets antioxidant transcription factor Nrf2 for ubiquitination by the Cullin 3-Roc1 ligase. Molecular and Cellular Biology, 25(1), 162-171.

Goodfellow, M. J., Borcar, A., Proctor, J. L., Greco, T., Rosenthal, R. E., \& Fiskum, G. (2020). Transcriptional activation of antioxidant gene expression by Nrf2 protects against mitochondrial dysfunction and neuronal death associated with acute and chronic neurodegeneration. Experimental Neurology, 328, 113247.

Guillin, O. M., Vindry, C., Ohlmann, T., \& Chavatte, L. (2019). Selenium, selenoproteins and viral infection. Nutrients, 11(9), 2101.

Hawkins, K. E., Joy, S., Delhove, J. M., Kotiadis, V. N., Fernandez, E., Fitzpatrick, L. M., Whiteford, J. R., King, P. J., Bolanos, J. P., Duchen, M. R., Waddington, S. N., \& McKay, T. R. (2016). NRF2 orchestrates the metabolic shift during induced pluripotent stem cell reprogramming. Cell Reports, 14(8), 1883-1891.

Held, J. M. (2020). Redox systems biology: Harnessing the sentinels of the cysteine redoxome. Antioxidants and Redox Signaling, 32(10), 659-676.

Hoffmann, P. R., \& Berry, M. J. (2008). The influence of selenium on immune responses. Molecular Nutrition and Food Research, 52(11), 1273-1280

Hou, J. C. (1997). Inhibitory effect of selenite and other antioxidants on complementmediated tissue injury in patients with epidemic hemorrhagic fever. Biological Trace Element Research, 56(1), 125-130.

Huang, H. C., Nguyen, T., \& Pickett, C. B. (2002). Phosphorylation of Nrf2 at Ser-40 by protein kinase $\mathrm{C}$ regulates antioxidant response element-mediated transcription. Journal of Biological Chemistry, 277(45), 42769-42774.

Israel, L. L., Braubach, O., Galstyan, A., Chiechi, A., Shatalova, E. S., Grodzinski, Z. Ding, H., Black, K. L., Ljubimova, J. Y., \& Holler, E. (2019). A combination of tri-Leucine and angiopep-2 drives a polyanionic polymalic acid nanodrug platform across the blood-brain barrier. ACS Nano, 13(2), 1253-1271.

Itoh, K., Wakabayashi, N., Katoh, Y., Ishii, T., Igarashi, K., Engel, J. D., \& Yamamoto, M. (1999). Keap1 represses nuclear activation of antioxidant responsive elements by Nrf2 through binding to the amino-terminal Neh2 domain. Genes and Development, 13(1), 76-86.

Jing, H., \& Lin, H. (2015). Sirtuins in epigenetic regulation. Chemical Reviews, $115(6), 2350-2375$

Jones, D. P., \& Sies, H. (2015). The redox code. Antioxidants and Redox Signaling, 23(9), 734-746

Jung, B. J., Yoo, H. S., Shin, S., Park, Y. J., \& Jeon, S. M. (2018). Dysregulation of NRF2 in cancer: From molecular mechanisms to therapeutic opportunities. Biomolecules and Therapeutics, 26(1), 57-68.

Kaidery, N. A., Ahuja, M., \& Thomas, B. (2019). Crosstalk between Nrf2 signaling and mitochondrial function in Parkinson's disease. Molecular and Cellular Neuroscience, 101, 103413.

Kalyanaraman, B., Cheng, G., Hardy, M., Ouari, O., Bennett, B., \& Zielonka, J. (2018). Teaching the basics of reactive oxygen species and their relevance to cancer biology: Mitochondrial reactive oxygen species detection, redox signaling, and targeted therapies. Redox Biology, 15, 347-362.

Kansanen, E., Kuosmanen, S. M., Leinonen, H., \& Levonen, A. L. (2013). The Keap1-Nrf2 pathway: Mechanisms of activation and dysregulation in cancer Redox Biology, 1(1), 45-49.

Katoh, Y., Iida, K., Kang, M. I., Kobayashi, A., Mizukami, M., Tong, K. I., \& Yamamoto, M. (2005). Evolutionary conserved N-terminal domain of Nrf2 is essential for the Keap1-mediated degradation of the protein by proteasome. Archives of Biochemistry and Biophysics, 433(2), 342-350.

Kim, S., Viswanath, A. N. I. Park, J. H., Lee, H. E., Park, A. Y., Choi, J. W, Kim, H., Londhe, A., Jang, B., Lee, J., Hwang. H., Lim, S., Pae, A., \& Park, K. (2020). Nrf2 activator via interference of Nrf2-Keap1 interaction has antioxidant and anti-inflammatory properties in Parkinson's disease animal model. Neuropharmacology, 167, 107989.

Kim, Y., \& Jang, H. H. (2019). Role of Cytosolic 2-Cys Prx1 and Prx2 in Redox Signaling. Antioxidants, 8(6), 169

Kobayashi, A., Kang, M. I., Okawa, H., Ohtsuji, M., Zenke, Y., Chiba, T., \& Yamamoto, M. (2004). Oxidative stress sensor Keapl functions as an adaptor for Cul3-based E3 ligase to regulate proteasomal degradation of Nrf2. Molecular and Cellular Biology, 24(16), 7130-7139.

Kobayashi, M., \& Yamamoto, M. (2005). Molecular mechanisms activating the Nrf2-Keap1 pathway of antioxidant gene regulation. Antioxidants and Redox Signaling, 7(3-4), 385-394.
Kopacz, A., Kloska, D., Forman, H. J., Jozkowicz, A., \& Grochot-Przeczek, A (2020). Beyond repression of Nrf2: An update on Keap1. Free Radical Biology and Medicine, 157, 63-74

Kosmachevskaya, O. V., Shumaev, K. B., \& Topunov, A. F. (2019). Electrophilic Signaling: The role of reactive carbonyl compounds. Biochemistry (Moscow), 84(1), 206-224

Kumar, A., \& Kumar, S. P. (2020). Biogenic selenium nanoparticles for their therapeutic application. Asian Journal of Pharmaceutical and Clinical Research, 13(1), 4-9.

Lee, D., Xu, I. M. J., Chiu, D. K. C., Leibold, J., Tse, A. P. W., Bao, M. H. R., Yuen, V. W.-H., Chan, C. Y., Lai, R. K.-H., Chin, D. W. C., Chan, D. F. F., Cheung, T. T., \& Chok, S. H. (2019). Induction of oxidative stress through inhibition of thioredoxin reductase 1 is an effective therapeutic approach for hepatocellular carcinoma. Hepatology, 69(4), 1768-1786.

Li, R., Jia, Z., \& Zhu, H. (2019). Regulation of Nrf2 signaling. Reactive Oxygen Species (Apex, NC), 24, 312-322.

Li, S., Bañuelos, G. S., Wu, L., \& Shi, W. (2014). The changing selenium nutritional status of Chinese residents. Nutrients, 6(3), 1103-1114.

Liu, P., Tian, W., Tao, S., Tillotson, J., Wijeratne, E. K., Gunatilaka, A. L., Zhang, D. D., \& Chapman, E. (2019). Non-covalent NRF2 activation confers greater cellular protection than covalent activation. Cell Chemical Biology, 26(10), $1427-1435$.

Lobanov, A. V., Hatfield, D. L., \& Gladyshev, V. N. (2009). Eukaryotic selenoproteins and selenoproteomes. Biochimica et Biophysica Acta - General Subjects, 1790(11), 1424-1428

Manoj, D., Saravanan, R., Santhanalakshmi, J., Agarwal, S., Gupta, V. K., \& Boukherroub, R. (2018). Towards green synthesis of monodisperse Cu nanoparticles: An efficient and high sensitive electrochemical nitrite sensor. Sensors and Actuators B: Chemical, 266, 873-882.

Marchiol, L. (2018). Nanotechnology in agriculture: New opportunities and perspectives. New Visions in Plant Science, 9, 121-132.

Marengo, B., Nitti, M., Furfaro, A. L., Colla, R., Ciucis, C. D., Marinari, U. M., Pronzato, M. A., Traverso, N., \& Domenicotti, C. (2016). Redox homeostasis and cellular antioxidant systems: Crucial players in cancer growth and therapy. Oxidative Medicine and Cellular Longevity, 2016, 6235641.

McMahon, M., Thomas, N., Itoh, K., Yamamoto, M., \& Hayes, J. D. (2006). Dimerization of substrate adaptors can facilitate cullin-mediated ubiquitylation of proteins by a "Tethering" mechanism a two-site interaction model for the Nrf2Keapl complex. Journal of Biological Chemistry, 281(34), 24756-24768.

Misra, B. B., Langefeld, C., Olivier, M., \& Cox, L. A. (2019). Integrated omics: Tools, advances and future approaches. Journal of Molecular Endocrinology, 62(1), R21-R45.

Miyata, Y., Matsuo, T., Sagara, Y., Ohba, K., Ohyama, K., \& Sakai, H. (2017) A mini-review of reactive oxygen species in urological cancer: Correlation with NADPH oxidases, angiogenesis, and apoptosis. International Journal of Molecular Sciences, 18(10), 2214.

Nguyen, T., Sherratt, P. J., Huang, H. C., Yang, C. S., \& Pickett, C. B. (2003). Increased protein stability as a mechanism that enhances Nrf2-mediated transcriptional activation of the antioxidant response element. Degradation of $\mathrm{Nrf} 2$ by the 26 S proteasome. Journal of Biological Chemistry, 278(7), 4536-4541.

Pacitti, D., Wang, T., Martin, S. A. M., Sweetman, J., \& Secombes, C. J. (2014). Insights into the fish thioredoxin system: Expression profile of thioredoxin and thioredoxin reductase in rainbow trout (Oncorhynchus mykiss) during infection and in vitro stimulation. Developmental and Comparative Immunology, 42(2), 261-277.

Padmanabhan, B., Tong, K. I., Kobayashi, A., Yamamoto, M., \& Yokoyama, S. (2008). Structural insights into the similar modes of Nrf2 transcription factor recognition by the cytoplasmic repressor Keap1. Journal of Synchrotron Radiation, 15(3), 273-276.

Pajares, M., Cuadrado, A., \& Rojo, A. I. (2017). Modulation of proteostasis by transcription factor NRF2 and impact in neurodegenerative diseases. Redox Biology, 11, 543-553.

Pajares, M., Jiménez-Moreno, N., García-Yagüe, Á. J., Escoll, M., de Ceballos, M. L., Van Leuven, F., Rabanof, A., Yamamotog, M., Rojoa, A. I., \& Cuadrado, A. (2016). Transcription factor NFE2L2/NRF2 is a regulator of macroautophagy genes. Autophagy, 12(10), 1902-1916.

Panieri, E., \& Saso, L. (2019). Potential applications of NRF2 inhibitors in cance therapy. Oxidative Medicine and Cellular Longevity, 2019, 8592348

Panieri, E., Buha, A., Telkoparan-Akillilar, P., Cevik, D., Kouretas, D., Veskoukis, A Skaperda, Z., Tsatsakis, A., Wallace, D., Suzen, S., \& Saso, L. (2020b). Potential applications of NRF2 modulators in cancer therapy. Antioxidants, 9(3), 193.

Panieri, E. Telkoparan-Akillilar, P., Suzen, S. \& Saso, L. (2020a). The NRF2/KE AP1 axis in the regulation of tumor metabolism: Mechanisms and therapeutic perspectives. Biomolecules, 10(5), 791

Perillo, B., Di Donato, M., Pezone, A., Di Zazzo, E., Giovannelli, P., Galasso, G., Castoria, G., \& Migliaccio, A. (2020). ROS in cancer therapy: The bright side of the moon. Experimental and Molecular Medicine, 52(2), 192-203. 
Pinto, G., Radulovic, M., \& Godovac-Zimmermann, J. (2018). Spatial perspectives in the redox code-mass spectrometric proteomics studies of moonlighting proteins. Mass Spectrometry Reviews, 37(1), 81-100.

Pradedova, E. V., Nimaeva, O. D., \& Salyaev, R. K. (2017). Redox processes in biological systems. Russian Journal of Plant Physiology, 64(6), 822-832.

Qiao, L., Dou, X., Yan, S., Zhang, B., \& Xu, C. (2020). Biogenic selenium nanoparticles synthesized by Lactobacillus casei ATCC 393 alleviate diquat-induced intestinal barrier dysfunction in C57BL/6 mice through their antioxidant activity. Food and Function, 11(4), 3020-3031.

Rayman, M. P. (2012). Selenium and human health. The Lancet, 379(9822), 12561268

Robledinos-Antón, N., Fernández-Ginés, R., Manda, G., \& Cuadrado, A. (2019). Activators and inhibitors of NRF2: A review of their potential for clinical development. Oxidative Medicine and Cellular Longevity, 2019, 9372182.

Roy, K., Wu, Y., Meitzler, J. L., Juhasz, A., Liu, H., Jiang, G., Lu, J., Antony, S., \& Doroshow, J. H. (2015). NADPH oxidases and cancer. Clinical Science, 128(12), 863-875.

Saab-Rincon, G., \& Valderrama, B. (2009). Protein engineering of redox-active enzymes. Antioxidants and Redox Signaling, 11(2), 167-192.

Sadowska-Bartosz, I., \& Bartosz, G. (2018). Redox nanoparticles: Synthesis, properties and perspectives of use for treatment of neurodegenerative diseases. Journal of Nanobiotechnology, 16(1), 87.

Samant, R. S., Livingston, C. M., Sontag, E. M., \& Frydman, J. (2018). Distinct proteostasis circuits co-operate in nuclear and cytoplasmic protein quality control. Nature, 563, 407-411.

Shah, S. Z. A., Zhao, D., Hussain, T., Sabir, N., Mangi, M. H., \& Yang, L. (2018) p62-Keap1-NRF2-ARE pathway: A contentious player for selective targeting of autophagy, oxidative stress and mitochondrial dysfunction in prion diseases. Frontiers in Molecular Neuroscience, 11,310.

Sharma, D., Kanchi, S., \& Bisetty, K. (2019). Biogenic synthesis of nanoparticles: A review. Arabian Journal of Chemistry, 12(8), 3576-3600.

Shcherbakov, A. B., Zholobak, N. M., \& Ivanov, V. K. (2020). Biological, biomedical and pharmaceutical applications of cerium oxide. In: Cerium oxide $\left(\mathrm{CeO}_{2}\right)$ : Synthesis, properties and applications. Elsevier. Pp. 279-358

Shin, J. M., Lee, K. M., Lee, H. J., Yun, J. H., \& Nho, C. W. (2019). Physalin A regulates the $\mathrm{Nr} 2$ pathway through $\mathrm{ERK}$ and $\mathrm{p} 38$ for induction of detoxifying enzymes. BMC Complementary and Alternative Medicine, 19(1), 101

Singh, C. K., Chhabra, G., Ndiaye, M. A., Garcia-Peterson, L. M., Mack, N. J., \& Ahmad, N. (2018). The role of sirtuins in antioxidant and redox signaling. Antioxidants and Redox Signaling, 28(8), 643-661.

Snezhkina, A. V., Kudryavtseva, A. V., Kardymon, O. L., Savvateeva, M. V., Melnikova, N. V., Krasnov, G. S., \& Dmitriev, A. A. (2019). ROS generation and antioxidant defense systems in normal and malignant cells. Oxidative Medicine and Cellular Longevity, 2019, 6175804.

Snyder, G. H., Cennerazzo, M. J., Karalis, A. J., \& Locey, D. (1981). Electrostatic influence of local cysteine environments on disulfide exchange kinetics. Biochemistry, 20(23), 6509-6519.

Song, D., Cheng, Y., Li, X., Wang, F., Lu, Z., Xiao, X., \& Wang, Y. (2017). Biogenic nanoselenium particles effectively attenuate oxidative stress-induced intestinal epithelial barrier injury by activating the $\mathrm{Nr} 2$ antioxidant pathway. ACS Applied Materials and Interfaces, 9(17), 14724-14740.

Staurengo-Ferrari, L., Badaro-Garcia, S., Hohmann, M. S., Manchope, M. F., Zaninelli, T. H., Casagrande, R., \& Verri, W. A. (2019). Contribution of Nrf2 modulation to the mechanism of action of analgesic and anti-inflammatory drugs in pre-clinical and clinical stages. Frontiers in Pharmacology, 9, 1536.

Stefanson, A., \& Bakovic, M. (2014). Dietary regulation of Keap1/Nrf2/ARE pathway: Focus on plant-derived compounds and trace minerals. Nutrients, 6(9), 3777-3801.

Steinbrenner, H., Al-Quraishy, S., Dkhil, M. A., Wunderlich, F., \& Sies, H. (2015) Dietary selenium in adjuvant therapy of viral and bacterial infections. Advances in Nutrition, 6(1), 73-82.

Taguchi, K., \& Yamamoto, M. (2017). The KEAP1-NRF2 system in cancer. Frontiers in Oncology, 7, 85.

Tian, W., De La Vega, M. R., Schmidlin, C. J., Ooi, A., \& Zhang, D. D. (2018). Kelch-like ECH-associated protein 1 (KEAP1) differentially regulates nuclear factor erythroid-2-related factors 1 and 2 (NRF1 and NRF2). Journal of Biological Chemistry, 293(6), 2029-2040.

Tonelli, C., Chio, I. I. C., \& Tuveson, D. A. (2018). Transcriptional regulation by Nrf2. Antioxidants and Redox Signaling, 29(17), 1727-1745.

Tong, K. I., Katoh, Y., Kusunoki, H., Itoh, K., Tanaka, T., \& Yamamoto, M. (2006) Keap1 recruits Neh2 through binding to ETGE and DLG motifs: Characterization of the two-site molecular recognition model. Molecular and Cellular Biology, 26(8), 2887-2900.

Tong, K. I., Padmanabhan, B., Kobayashi, A., Shang, C., Hirotsu, Y., Yokoyama, S. \& Yamamoto, M. (2007). Different electrostatic potentials define ETGE and DLG motifs as hinge and latch in oxidative stress response. Molecular and Cellular Biology, 27(21), 7511-7521.
Tsekhmistrenko, O. S., Bityutskyy, V. S., Tsekhmistrenko, S. I., Kharchishin, V. M. Melnichenko, O. M., Rozputnyy, O. I., Malina, V. V., Prysiazhniuk, N. M., Melnichenko, Y. O., Vered, P. I., Shulko, O. P., \& Onyshchenko L. S. (2020c). Nanotechnologies and environment: A review of pros and cons. Ukrainian Journal of Ecology, 10(3), 162-172.

Tsekhmistrenko, O. S., Tsekhmistrenko, S. I., Bityutskyy, V. S., Melnichenko, O. M., \& Oleshko, O. A. (2018). Biomimetic and antioxidant activity of nanocrystalline cerium dioxide. World of Medicine and Biology, 14(63), 196-201.

Tsekhmistrenko, O., Bityutskyy, V., Tsekhmistrenko, S., Melnichenko, O., Tymoshok, N., \& Spivak, M. (2019). Use of nanoparticles of metals and non-metals in poultry farming. Animal Husbandry Products Production and Processing, 2 , 113-130.

Tsekhmistrenko, S. I., \& Polishchuk, V. M. (2010). Vikovi osoblyvosti funktsionuvannia systemy antyoksydantnoho zakhystu krovi strausiv [Age specifics of functioning of the antioxidant defense system in the blood of ostriches]. Ukrainskyi Biokhimichnyi Zhurnal, 82(5), 92-97 (in Ukrainian).

Tsekhmistrenko, S. I., \& Ponomarenko, N. V. (2013). Sklad lipidiv ta yikh peroksydne okyslennia u pidshlunkovii zalozi perepeliv za dii nitrativ i u razi zghodovuvannia nasinnia amarantu [Lipid content and peroxidation in the pancreas of quails under the effect of nitrates and in cases of feeding with seeds of amaranth]. Ukrainskyi Biokhimichnyi Zhurnal, 85(2), 84-92 (in Ukrainian).

Tsekhmistrenko, S. I., Bityutskyy, V. S., \& Tsekhmistrenko, O. S. (2020b). Markers of oxidative stress in the blood of quails under the influence of selenium nanoparticles. Impact of modernity on science and practice. Abstracts of XVIII International Scientific and Practical Conference. Boston. Pp. 177-180.

Tsekhmistrenko, S. I., Bityutskyy, V. S., Tsekhmistrenko, O. S., Horalskyi, L. P., Tymoshok, N. O., \& Spivak, M. Y. (2020). Bacterial synthesis of nanoparticles: A green approach. Biosystems Diversity, 28(1), 9-17.

Tsekhmistrenko, S. I., Bityutskyy, V. S., Tsekhmistrenko, O. S., Melnichenko, O. M., Kharchyshyn, V. M., Tymoshok, N. O., Ponomarenko, N. V., Polishchuk, S. A., Rol, N. V., Fedorchenko, M. M., Melnichenko, Y. O., Merzlova, H. V., Shulko, O. P., \& Demchenko, A. A. (2020a). Effects of selenium compounds and toxicant action on oxidative biomarkers in quails. Ukrainian Journal of Ecology, 10(2), 232-239.

Tu, W., Wang, H., Li, S., Liu, Q., \& Sha, H. (2019). The anti-inflammatory and antioxidant mechanisms of the Keap1/Nrf2/ARE signaling pathway in chronic diseases. Aging and disease, 10(3), 637-651.

Uruno, A., \& Motohashi, H. (2011). The Keap1-Nrf2 system as an in vivo sensor for electrophiles. Nitric Oxide, 25(2), 153-160.

Villeneuve, N. F., Lau, A., \& Zhang, D. D. (2010). Regulation of the Nrf2-Keap1 antioxidant response by the ubiquitin proteasome system: an insight into cullinring ubiquitin ligases. Antioxidants and Redox Signaling, 13(11), 1699-1712.

Wang, T., Yang, L., Zhang, B., \& Liu, J. (2010). Extracellular biosynthesis and transformation of selenium nanoparticles and application in $\mathrm{H}_{2} \mathrm{O}_{2}$ biosensor. Colloids and Surfaces B: Biointerfaces, 80(1), 94-102.

Wang, X. J., Sun, Z., Villeneuve, N. F., Zhang, S., Zhao, F., Li, Y., Chen, W., Yi, X. Zheng, W., Wondrak, G. T., Wong, P. K., \& Wong, P. K. (2008). Nrf2 enhances resistance of cancer cells to chemotherapeutic drugs, the dark side of Nrf2. Carcinogenesis, 29(6), 1235-1243.

Wang, Z., Li, Q., Chen, Y., Cui, B., Li, Y., Besenbacher, F., \& Dong, M. (2018). The ambipolar transport behavior of WSe 2 transistors and its analogue circuits. NPG Asia Materials, 10(8), 703-712.

Wen, Z., Liu, W., Li, X., Chen, W., Liu, Z., \& Wen, J. (2019). A protective role of the NRF2-Keapl pathway in maintaining intestinal barrier function. Oxidative Medicine and Cellular Longevity, 2019, 1759149.

Xia, Y., Zhao, Y., Zhang, F., Chen, B., Hu, X., Weir, M. D., Schneider, A., Jia, L., Gu, N., \& Xu, H. H. (2019). Iron oxide nanoparticles in liquid or powder form enhanced osteogenesis via stem cells on injectable calcium phosphate scaffold. Nanomedicine: Nanotechnology, Biology and Medicine, 21, 102069.

Xiao, X., Song, D., Cheng, Y., Hu, Y., Wang, F., Lu, Z., \& Wang, Y. (2019). Biogenic nanoselenium particles activate Nrf2-ARE pathway by phosphorylating p38, ERK1/2, and AKT on IPEC-J2 cells. Journal of Cellular Physiology, 234(7), 11227-11234

Xu, C., Guo, Y., Qiao, L., Ma, L., Cheng, Y., \& Roman, A. (2018). Biogenic synthesis of novel functionalized selenium nanoparticles by Lactobacillus casei ATCC 393 and its protective effects on intestinal barrier dysfunction caused by enterotoxigenic Escherichia coli K88. Frontiers in Microbiology, 9, 1129.

Yamamoto, M., Kensler, T. W., \& Motohashi, H. (2018). The KEAP1-NRF2 system: A thiol-based sensor-effector apparatus for maintaining redox homeostasis. Physiological Reviews, 98(3), 1169-1203.

Yang, Y., Bazhin, A. V., Werner, J., \& Karakhanova, S. (2013). Reactive oxygen species in the immune system. International Reviews of Immunology, 32(3), 249-270.

Yoshida, K., \& Hisabori, T. (2016). Two distinct redox cascades cooperatively regulate chloroplast functions and sustain plant viability. Proceedings of the National Academy of Sciences, 113(27), E3967-E3976.

Yu, R., Mandlekar, S., Lei, W., Fahl, W. E., Tan, T. H., \& Kong, A. N. T. (2000). p38 mitogen-activated protein kinase negatively regulates the induction of phase 
II drug-metabolizing enzymes that detoxify carcinogens. Journal of Biological Chemistry, 275(4), 2322-2327.

Zenkov, N. K., Menshchikova, E. B., \& Tkachev, V. O. (2013). Keap1/Nrf2/ARE redox-sensitive signaling system as a pharmacological target. Biochemistry (Moscow), 78(1), 19-36.

Zhang, D. D., \& Hannink, M. (2003). Distinct cysteine residues in Keapl are required for Keap1-dependent ubiquitination of Nrf2 and for stabilization of $\mathrm{Nrf} 2$ by chemopreventive agents and oxidative stress. Molecular and Cellular Biology, 23(22), 8137-8151.

Zhang, D. D., Lo, S. C., Cross, J. V., Templeton, D. J., \& Hannink, M. (2004). Keap1 is a redox-regulated substrate adaptor protein for a Cul3-dependent ubiquitin ligase complex. Molecular and Cellular Biology, 24(24), 10941-10953.

Zhang, J., Taylor, E. W., Bennett, K., Saad, R., \& Rayman, M. P. (2020). Association between regional selenium status and reported outcome of COVID-19 cases in China. The American Journal of Clinical Nutrition, 111(6), 1297-1299.
Zhao, J., \& Zeng, Z. (2020). Combined effects of AKT serine/threonine kinase 1 polymorphisms and environment on congenital heart disease risk: A casecontrol study. Medicine, 99(26), e20400.

Zhao, L., Cox, A. G., Ruzicka, J. A., Bhat, A. A., Zhang, W., \& Taylor, E. W. (2000). Molecular modeling and in vitro activity of an HIV-1-encoded glutathione peroxidase. Proceedings of the National Academy of Sciences, 97(12), 6356-6361.

Zhao, R. Z., Jiang, S., Zhang, L., \& Yu, Z. B. (2019). Mitochondrial electron transport chain, ROS generation and uncoupling. International Journal of Molecular Medicine, 44(1), 3-15.

Zheng, F., \& Li, H. (2019). Evaluation of Nrf2 with exposure to nanoparticles. In: Nanotoxicity. Humana Press, New York. Pp. 229-246.

Zollman, S., Godt, D., Prive, G. G., Couderc, J. L., \& Laski, F. A. (1994). The BTB domain, found primarily in zinc finger proteins, defines an evolutionarily conserved family that includes several developmentally regulated genes in Drosophila. Proceedings of the National Academy of Sciences, 91(22), 10717-10721. 